\title{
Investigating the Role of Hydroalcoholic Extract of Apium graveolens and Cinnamon zeylanicum on Metabolically Change and Ovarian Oxidative Injury in a Rat Model of Polycystic Ovary Syndrome
}

\author{
Fariba Khodaeifar ${ }^{1}$, Seyyed Mohammad Bagher Fazljou ${ }^{1}$, Arash Khaki ${ }^{2}$ Mohammadali Torbati ${ }^{3}$ Elahe \\ Olad Saheb Madarek ${ }^{2}$, Amir Afshin Khaki ${ }^{4^{*} \mathbb{D}}$, Majid Shokoohi ${ }^{4,5}$, Amir Hossein Dalili ${ }^{6}$
}

\begin{abstract}
Objectives: The present study aimed to compare the role of the hydroalcoholic extract of Apium graveolens and Cinnamon zeylanicum on metabolically change and ovarian oxidative injury in a rat model of polycystic ovary syndrome (PCOS).

Materials and Methods: In this experimental research, 64 female Wistar rats with breeding were used including the following groups (G): (I) G1: healthy control; (II) G2: PCOS which received no therapy; (III) G3: PCOS + A. graveolens; (IV) G4: A. graveolens; (V) G5: PCOS + C. zeylanicum; (VI) G6: no PCOS + C. zeylanicum; (VII) G7: PCOS + C. zeylanicum and A. graveolens; and (VIII) G8: C. zeylanicum and A. graveolens. The PCOS was induced by a single dose of the intramuscularly injected estradiol valerate (16 mg/ $\mathrm{kg}$ ). After 14 days, the animals were anesthetized, then their plasma samples were used to check the blood sugar (BS), insulin, and lipid profile. The ovaries of the rats were removed and fixed for histopathological assessment. In addition, the oxidative stress marker in ovarian tissue was evaluated.

Results: The levels of BS, insulin, and lipid profile in plasma significantly enhanced in G2 $(P<0.05)$ while decreasing significantly in the therapy groups, $(P<0.05)$. Moreover, a significant decline was observed in the serum level of high-density lipoprotein (HDL) in G2 $(P<0.05)$ while it enhanced significantly in the therapeutic animals $(P<0.05)$. Furthermore, a negative change was found in the PCOS group on the ovarian tissue. Besides the oxidative stress enhanced in this tissue while in the treated groups this change was improved.

Conclusions: Generally, it was revealed that the extract of A. graveolens and C. zeylanicum had a useful impact on regulating the serum levels of fast blood sugar (FBS), insulin, lipid profile, and oxidative stress markers in the palliation of the PCOS complications. Keywords: Apium graveolens, Cinnamon zeylanicum, PCOS, Lipid profile
\end{abstract}

\begin{abstract}
Introduction
Polycystic ovary syndrome (PCOS) is known as a publicized derangement of the endocrine system in women who can be fertilized $(1,2)$. Other complications of this syndrome in long-term consist of type 2 diabetes, insulin resistance syndrome, hypertension, and cardiovascular diseases. In addition, the risk of hyperplasia and cancer of the endometrium is higher in women with PCOS who do not receive any treatment (3). In the PCOS patients and hyperandrogenism, there is an extensive attachment between the hyperinsulinemia and hyperandrogenism as the level of insulin shows an intense correlation with the exclusion of the steroid from the adrenal (4). Besides, in these patients, hyperinsulinemia is accompanied by
\end{abstract}

elevated levels of low-density lipoprotein (LDL), very low-density lipoprotein (VLDL), triglyceride, total cholesterol (5), and a decline in the serum level of highdensity lipoprotein (HDL) (6). In addition to resistance to insulin, the lipid metabolism of the females with PCOS is probably transformed by ovarian or adrenal secretion of the sex steroid hormones. The effect of sex steroids on fat metabolism that involved the actions of estrogens and androgens is complicated in this scenario $(7,8)$.

Using medicinal plants to treat female sexual disorders in Iran has a broad background and studies have shown that the use of these plants has a positive effect on treating metabolic diseases including diabetes and PCOS $(9,10)$.

Apium graveolens includes a lot of antioxidant

Received 2 March 2018, Accepted 22 November 2018, Available online 15 December 2018

${ }^{1}$ Department of Traditional Medicine, Faculty of Traditional Medicine, Tabriz University of Medical Sciences, Tabriz, Iran. ${ }^{2}$ Women's Reproductive Health Research Center, Tabriz University of Medical Sciences, Tabriz, Iran. ${ }^{3}$ Department of Food Science and Technology, Faculty of Pharmacy, Tabriz University of Medical Sciences, Tabriz, Iran. ${ }^{4}$ Department of Anatomical Sciences, Faculty of Medicine, Tabriz University of Medical Sciences, Tabriz, Iran. ${ }^{5}$ Student Research Committee, Tabriz University of Medical Sciences, Tabriz, Iran. ${ }^{6}$ Student Research Committee, Faculty of Medicine, Tabriz University of Medical Sciences, Tabriz, Iran.

*Corresponding Author: Amir Afshin Khaki, Department of Anatomical Sciences, Faculty of Medicine, Tabriz University of Medical Sciences (TBZMED), Tabriz, Iran. Tel: +989144157161, Email: dr.aakhaki@yahoo.com 
compounds such as vitamin $\mathrm{C}$, which reduce the edema of the body cells, and also eliminate and act against rheumatism and cancerous diseases (11). The A. graveolens extract has the potential to interdict the activity of free radicals and stopper of lipid peroxidation. Accordingly, this plant has antioxidant and anti-diabetes confidants. Additionally, previous studies showed that the extract of this herb is anti-inflammatory and analgesic and can decrease cholesterol and triglyceride $(12,13)$.

The skin of Cinnamon zeylanicum includes 0.5\%-2.5\% essential oil. Besides, it comprises more than fifty various combinations of which $65 \%-80 \%$ is aldehyde cinematic. The major components of cinnamon are aldehyde (65\%$80 \%)$ and eugenol (10\%) which have high antioxidant activities and antibacterial properties (14).

As a result, given that the effects of these herbs have not been studied on PCOS, the present research sought to test the impact of C. zeylanicum and A. graveolens extracts on the ovarian oxidative damage and metabolically change in rats altered due to the PCOS.

\section{Materials and Methods}

In this research, 64 female Wistar rats weighing 200-250 $\mathrm{g}$ were used. All the animals were purchased from Razi Institute of Mashhad and were intently kept in the animal laboratory under standard conditions (Temperature of $22^{\circ} \mathrm{C} \& 12 / 12$ hours of the light-dark cycle) and had access to food and water.

\section{Study Design and Groups}

All the rats were divided into 8 groups as follows:

1. Healthy control group: daily oral intake of physiological serum

2. PCOS group: received no treatment

3. PCOS group that received therapy using the $A$. graveolens $(200 \mathrm{mg} / \mathrm{kg})$ extract for 14 days, orally (PAG)

4. Sham group: including 8 rats that were orally treated with the A. graveolens $(200 \mathrm{mg} / \mathrm{kg}$ ) extract for 14 days, without PCOS induction (AG)

5. PCOS group that received therapy with $C$. zeylanicum (200 mg/kg) extract for 14 days, orally. (PCA)

6. Sham group: including 8 rats that were treated with the C. zeylanicum $(200 \mathrm{mg} / \mathrm{kg})$ extract for 14 days without PCOS induction (CA)

7. PCOS group that received therapy with the $C$. zeylanicum $(200 \mathrm{mg} / \mathrm{kg}$ ) and A. graveolens $(200 \mathrm{mg} /$ $\mathrm{kg})$ extracts for 14 days $(\mathrm{PCO}+\mathrm{C}+\mathrm{A})$

8. No PCOS + C. zeylanicum and A. graveolens $(\mathrm{C}+\mathrm{A})$.

The PCOS was induced by estradiol valerate with a single dose $(16 \mathrm{mg} / \mathrm{kg})$ which was intramuscularly injected (Sigma, Germany) and solved in 200 lambdas of sesame oil (7). The smear of the vagina was daily examined and confirmed the estrus cycle in PCOS rats. After the therapy, all the animals were anesthetized using ketamine and xylazine $(5 / 1 \mathrm{mg} / \mathrm{kg})(15)$. Then, the blood samples were derived from the inferior vena cava and the serum was separated by centrifuge at $3000 \mathrm{~g}$. The taken serum was kept at $-70^{\circ} \mathrm{C}$ for further assessment.

Preparation of Apium graveolens and Cinnamon zeylanicum Hydroalcoholic Extracts

To provide the A. graveolens and C. zeylanicum extracts, $0.5 \mathrm{~kg}$ of each plant was first powdered, then solved in one liter of ethanol $50 \%$ and kept on a shaker (Thermo Fisher) at the room temperature for 48 hours. Next, the dilution was filtered and centrifuged at $3000 \mathrm{rpm}$ for 10 minutes. Afterward, the solution was infused into a dish to vaporize the solvent. And finally, to attain a suitable concentration, the obtained extract was solved in sterile physiological serum.

Assessing Plasma Glucose, HDL, LDL, and Cholesterol Levels

At the end of the study, serum glucose, HDL, LDL, triglyceride (TG), and cholesterol levels were defined by mercantile kits (Parsazmun, Iran). The quantity was expressed as $\mathrm{mg} / \mathrm{dL}$.

Assay of Serum Insulin Level

Insulin levels in plasma were measured by a method based on enzyme-linked immunosorbent assay (ELISA) using the kit of Rat Insulin (Mercodia).

Assessing Oxidative Stress Markers in the Ovarian Tissue To evaluate the markers of oxidative stress in the tissue, the ovarian tissues were homogenized. Malondialdehyde (MDA) level was assessed to investigate the lipid peroxidation in the ovary as well. In order to provide a solution of TBA-TCA-HCL, $375 \mathrm{mg}$ of thiobarbituric acid (TBA) was solved in $2000 \mu \mathrm{L}$ of HCL (hydrochloric acid). Next, it was added to $100 \mathrm{~mL}$ of the trichloroacetic acid (TCA) 15\%. To complete the resolution of the sediment, water bath with a temperature of $50^{\circ} \mathrm{C}$ was used. Then, the ovarian tissue was weighed and immediately homogenized with potassium chloride $5.1 \%$ to create $10 \%$ homogenized admixture. Subsequently, 1000 lambdas of homogenized tissue mixture was dissolved in two ccs of TBA-TCA$\mathrm{HCl}$ solution, afterward, it was put in the boiling water for 45 minutes to heat in order to obtain a pink-orange solution. After cooling the solution, it was centrifuged at $1000 \mathrm{rpm}$ for 10 minutes. The absorption was read by a spectrophotometer. The superoxide dismutase (SOD) and glutathione peroxidase (GPX) levels were recognized in the ovarian tissue by an ELISA reader device (Antus) conforming the protocols of the kits (Randox, \& Ransod, UK) (16).

Histological Assessment

After euthanasia, the ovary tissues were accumulated 
from each rat. Then, the tissues were fixed in the $10 \%$ neutral-buffered formalin followed by embedding in paraffin. They were then divided into $5 \mu \mathrm{m}$ sectioned, deparaffinized, stained by hematoxylin \& eosin staining (H\&E), and dehydrated in ethanol 95\%, 90\%, and 70\%. Afterward, they were cleared by the xylene. The sections were observed by a microscope (Olympus).

Statistical Analysis

All the data were analyzed by the SPSS software, version 19 (USA). Moreover, the data were presented as mean \pm SEM (standard error of the mean) and were compared using one-way ANOVA and Tukey post hoc test. The level of $P<0.05$ was considered significant.

\section{Results}

Plasma FBS and Insulin Level

Based on Figure 1, the plasma level of FBS in the PCOS group was significantly higher than that of the control $(P$
$<0.001)$. Besides, the plasma glucose level in the treated groups that received $A$. graveolens and $C$. zeylanicum extracts was lower than that of the PCO group $(P<$ $0.05)$. Figure 2 shows that the plasma level of insulin was statistically higher in the PCOS group compared to that of the control group $(P<0.05)$. Alternatively, a significant decline was found the insulin level of plasma in groups that were treated with $A$. graveolens and C. zeylanicum extracts as compared to that of the PCOS group $(P<0.05)$.

\section{Lipid Profile \\ Plasma Cholesterol Level}

The cholesterol level in plasma significantly enhanced in the PCOS group when compared with that of the control $(P<0.05)$. However, the plasma cholesterol level declined in all those groups that were treated with $A$. graveolens and C. zeylanicum extracts as compared with that of the control group $(P<0.05)$ (Table 1$)$

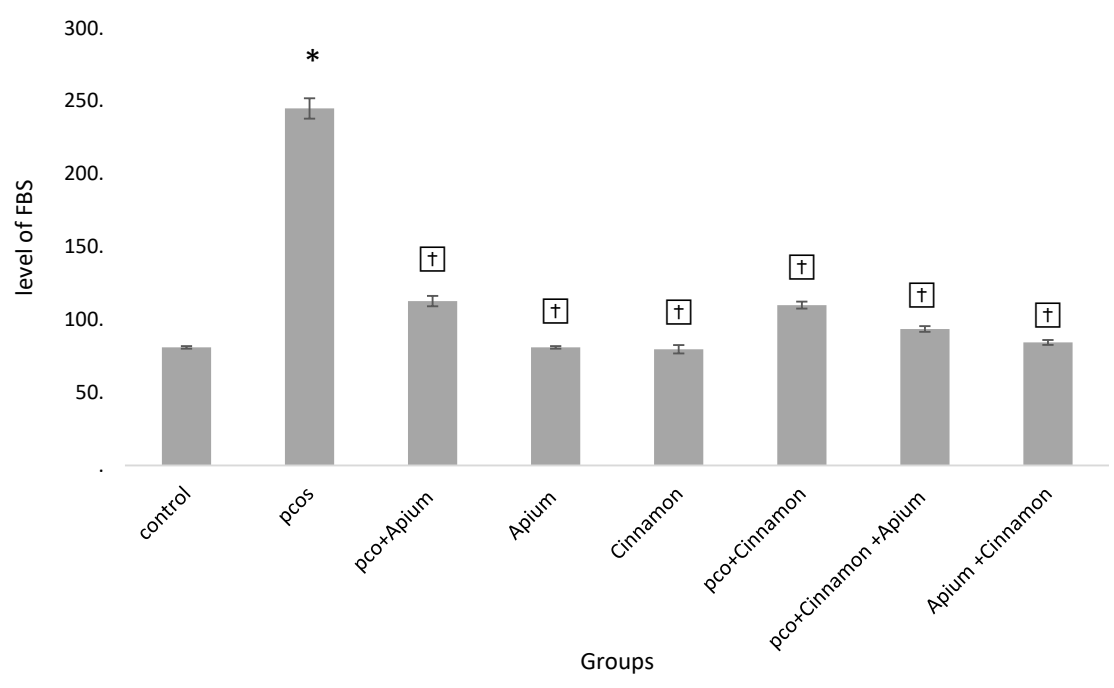

Figure 1. Serum Level of FBS. The asterisk * shows significant difference with control group and the symbol of $\dagger$ means the significant difference with PCO group.

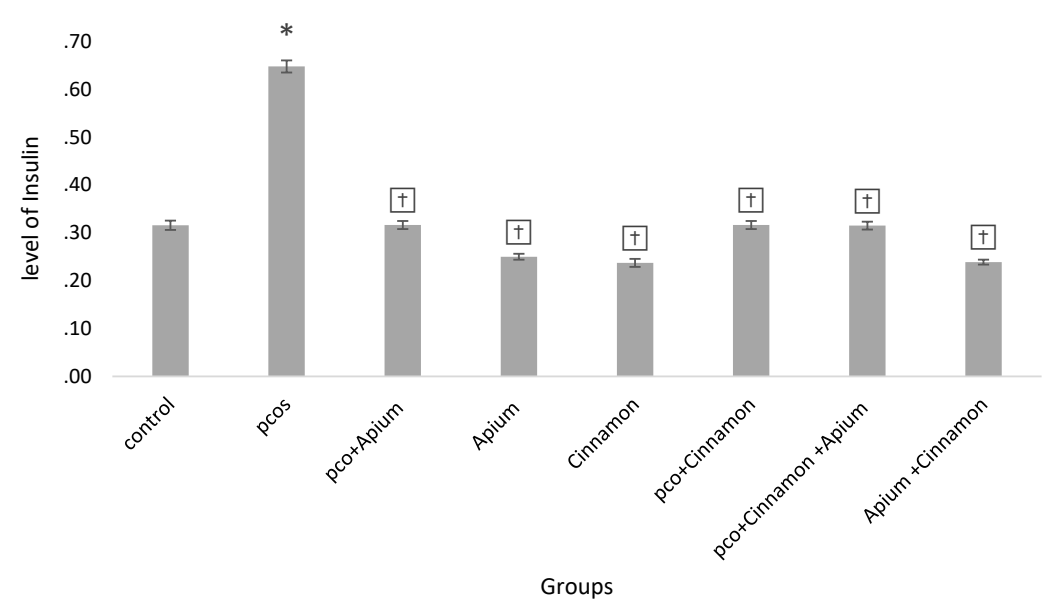

Figure 2. Serum Level of Insulin. The asterisk * shows significant difference with control group and the symbol of $\dagger$ means the significant difference with PCO group. 
Table 1. Comparing the Serum Level of Cholesterol, HDL, LDL, and TG in the Study Groups

\begin{tabular}{|c|c|c|c|c|}
\hline Group & Cholesterol (mg/dL) & $\mathrm{HDL}(\mathrm{mg} / \mathrm{dL})$ & LDL (mg/dL) & $\mathrm{TG}$ (mg/dL) \\
\hline Control & $68.050 \pm 2.32$ & $45.694 \pm 1.59$ & $11.144 \pm 1.51$ & $35.215 \pm 1.58$ \\
\hline PCOS & $80.989 \pm 1.04^{b}$ & $28.922 \pm 0.53^{b}$ & $23.228 \pm 3.68$ & $60.372 \pm 4.58^{b}$ \\
\hline PGA & $70.5726 \pm 3.37^{a}$ & $40.213 \pm 1.15^{\mathrm{a}}$ & $15.912 \pm 2.03^{\mathrm{a}}$ & $40.780 \pm 4.13^{\mathrm{a}}$ \\
\hline AG & $69.526 \pm 3.37^{a}$ & $42.213 \pm 1.15^{\mathrm{a}}$ & $10.052 \pm 2.03^{a}$ & $36.789 \pm 4.13^{a}$ \\
\hline PCZ & $72.726 \pm 3.37^{a}$ & $39.213 \pm 1.15^{a}$ & $16.952 \pm 2.03^{\mathrm{a}}$ & $38.895 \pm 4.13^{a}$ \\
\hline $\mathrm{CZ}$ & $67.050 \pm 2.32$ & $43.894 \pm 1.59$ & $10.174 \pm 1.51$ & $35.565 \pm 1.58$ \\
\hline $\mathrm{PCO}+\mathrm{C}+\mathrm{A}$ & $66.576 \pm 3.37^{a}$ & $42.413 \pm 1.15^{a}$ & $14.152 \pm 2.03^{\mathrm{a}}$ & $42.785 \pm 4.13^{a}$ \\
\hline$C+A$ & $67.150 \pm 2.32$ & $46.894 \pm 1.59$ & $11.474 \pm 1.51$ & $37.505 \pm 1.58$ \\
\hline
\end{tabular}

Note. Control: Control group; PCOS: PCO (polycystic ovary syndrome) group that received normal saline by oral gavage; PGA: PCOS group that were treated with hydroalcoholic extract of Apium (A.) graveolens (200 mg/kg); AG: Healthy group which received the hydroalcoholic extract of $A$. graveolens (200 mg/kg); PCA: PCOS group that received therapy with C. zeylanicum extract ( $200 \mathrm{mg} / \mathrm{kg} / \mathrm{orally})$; CA: Healthy group which received the hydroalcoholic extract of C. zeylanicum (200 mg/kg); PCO+C+A: PCOS group that received therapy with C. zeylanicum (200 mg/kg) and A. graveolens $(200 \mathrm{mg} / \mathrm{kg})$ extracts; C+A: No PCOS + C. zeylanicum and A. graveolens.

${ }^{a}$ A substantial difference with the control group; ${ }^{b}$ The notable difference with PCOS group.

Serum Level of Low-Density Lipoprotein

The LDL plasma level also increased in the PCOS group compared to that of the control group $(P>0.05)$. In addition, a significant decline was observed in the plasma LDL level among the therapy groups that received $A$. graveolens and C. zeylanicum extracts compared to that of the PCOS group $(P<0.05)$ (Table 1$)$.

Plasma Level of High-Density Lipoprotein

The level of HDL in plasma significantly decreased in the PCOS group when compared with that of the control group $(P<0.05)$. However, in the therapy groups, it enhanced significantly compared to that of the PCOS group $(P<0.05)$ (Table 1$)$

Plasma Level of Triglycerides

The TG level in plasma a significant increase was detected

Table 2. The Markers of Oxidative Stress in Ovarian Tissue

\begin{tabular}{lccc}
\hline Groups & MDA \pm SD & SOD \pm SD & GPx \pm SD \\
\hline Control & $80 \pm 6.5$ & $1.63 \pm 0.21$ & $33 \pm 3.21$ \\
PCOS & $140 \pm 7.35^{\mathrm{b}}$ & $0.60 \pm 0.11^{\mathrm{b}}$ & $14.35 \pm 2.32^{\mathrm{b}}$ \\
PGA & $100 \pm 5.7^{\mathrm{a}}$ & $0.96 \pm 0.18^{\mathrm{a}}$ & $26.75 \pm 3.6^{\mathrm{a}}$ \\
AG & $825.25 \pm^{\mathrm{a}}$ & $1.57 \pm 0.24^{\mathrm{a}}$ & $33.65 \pm 3.25^{\mathrm{a}}$ \\
PCZ & $105 \pm 10.2^{\mathrm{a}}$ & $1.06 \pm 0.15^{\mathrm{a}}$ & $27.56 \pm 4.2^{\mathrm{a}}$ \\
CZ & $79 \pm 3.5$ & $1.65 \pm 0.25^{\mathrm{a}}$ & $34.65 \pm 2.25^{\mathrm{a}}$ \\
PCO+C+A & $95 \pm 8.4$ & $1.18 \pm 0.15^{\mathrm{a}}$ & $27.25 \pm 4.05^{\mathrm{a}}$ \\
C+A & $75 \pm 3.2$ & $1.70 \pm 0.25^{\mathrm{a}}$ & $35.05 \pm 3.15^{\mathrm{a}}$ \\
\hline
\end{tabular}

Note. Control: Control group; PCOS: PCO group that received normal saline by oral gavage; PGA: PCOS group treated with hydroalcoholic extract of Apium graveolens $(200 \mathrm{mg} / \mathrm{kg}) ; \mathrm{AG}$ : Healthy group which received the hydroalcoholic extract of $A$. graveolens (200 mg/kg); PCA: PCOS group that received therapy with C. zeylanicum extract $(200 \mathrm{mg}$ / $\mathrm{kg} /$ orally); CA: Healthy group received the hydroalcoholic extract of C. zeylanicum ( $200 \mathrm{mg} / \mathrm{kg}$ ); $\mathrm{PCO}+\mathrm{C}+\mathrm{A}$ : The PCOS group that received therapy with C. zeylanicum $(200 \mathrm{mg} / \mathrm{kg})$ and $A$. graveolens $(200 \mathrm{mg} / \mathrm{kg})$ extracts; C+A: No PCOS + C. zeylanicum and $A$. graveolens.

${ }^{\mathrm{a}} \mathrm{A}$ substantial difference with the control group; ${ }^{\mathrm{b}}$ The notable difference with PCOS group. in the PCOS group compared with that of the control ( $P$ $<0.05)$. In addition, there was a significant decline in the serum level of TG in the groups treated with A. graveolens and C. zeylanicum extracts compared to TG serum level of the PCOS group $(P<0.05)$ (Table 2$)$

\section{Oxidative Stress Markers in Ovarian Tissue}

A significant level of MDA was observed in group 2 as compared to the control group $(P<0.05)$. However, in therapy groups that received $A$. graveolens and C. zeylanicum extracts, separately and together, the malondialdehyde level in ovarian tissue declined significantly $(P<0.05)$. The SOD and GPX levels in ovarian tissue showed a significant decrease in group 2, $(P<0.05)$. However, in therapeutic groups, it increased significantly compared to that of the group $2(P<0.05)$.

\section{Histological Changes}

The histological assessment showed a normal histology of the ovarian tissue in the control group. Besides, the number of follicles was normal in this group. However, in the PCOS group, PCOS caused damage to the ovarian tissue and the number of normal follicles decreased in this group as well. In addition, the atretic follicles were observed in the ovarian tissue of the rats with PCOS. Moreover, treatment with A. graveolens and C. zeylanicum extracts was found to decrease the number of atretic follicles while enhancing the normal follicles (Figure 3).

\section{Discussion}

The results indicated that the PCOS led to an increase in the plasma level of the FBS and insulin. In this context, recent research has shown that the PCOS can induce the metabolic syndrome. Besides, it mostly depends on insulin resistance attended by compensatory hyperinsulinemia, resulting in an enhanced risk for the expansion of type 2 diabetes mellitus $(17,18)$. Moreover, the results also showed that the A. graveolens and C. zeylanicum extracts 


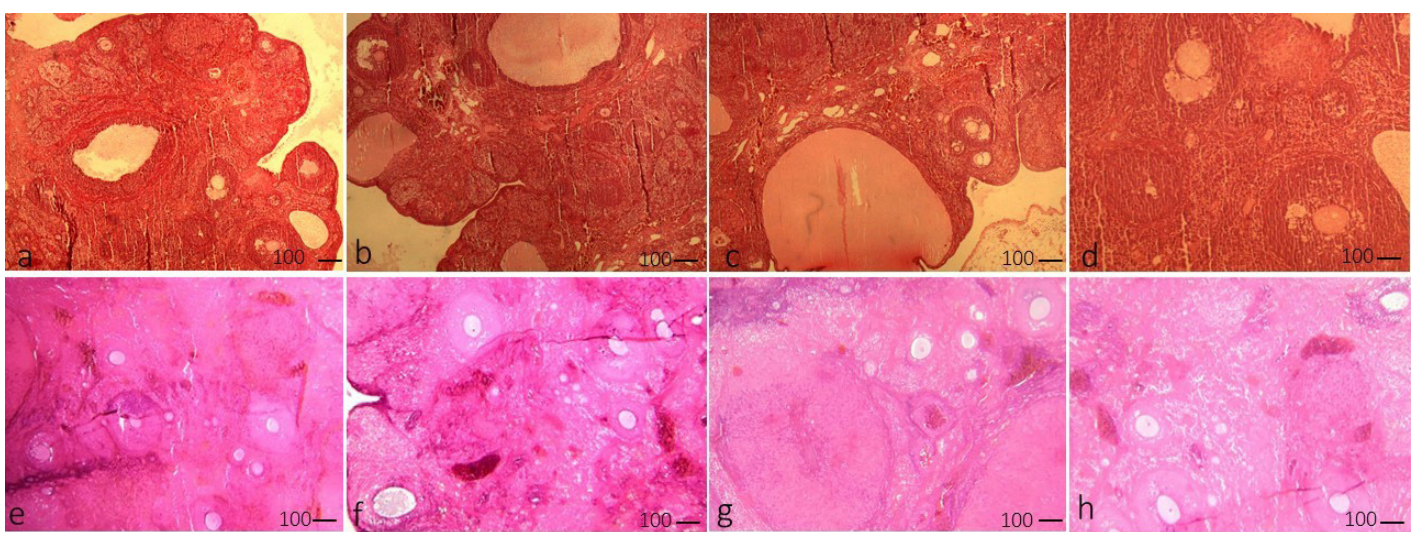

Figure. 3. The Histological Findings in Studied Groups. a: control group; b: PCO group that intake normal saline by oral gavage; c: PCOS group treated with hydroalcoholic extract of Apium graveolens (200 mg/kg); d: healthy group intake the hydroalcoholic extract of Apium graveolens (200 mg/kg); e: PCOS group that received therapy with extract of Cinnamon Zeylanicum ( $200 \mathrm{mg} / \mathrm{kg} / \mathrm{orally})$; f: healthy group intake the hydroalcoholicextract of Cinnamon zeylanicum (200 mg/kg), g: PCOS group that received therapy with extract of Cinnamon Zeylanicum (200 mg/kg) and Apium graveolens (200 mg/kg); h: No PCOS + Cinnamon zeylanicum and Apium graveolens.

significantly affected the plasma levels of fast blood sugar (FBS) and insulin. The antioxidant properties of this plant that prevented the oxygen species activity and reduced the oxidative stress may be a justification for this result. Gutierrez et al indicated that administering A. graveolens extract could decrease the level of FBS in plasma of the diabetic rat (19). The decline in insulin level by A. graveolens extract may stem from the presence of compounds such as luteolin flavonoids and other compounds such as apoysyl glucoside 7-O., Apigenin 7-O apysyl glucoside, and Cryosurial 7-O apysyl glucoside (20). Notably, the reduction in body capacity of antioxidant has been shown to have a connection with the insulin resistance (21). Furthermore, the C. zeylanicum extract could decrease the plasma level of FBS and insulin. Besides, it reduced the insulin resistance which is feasible due to the presence of an antioxidant combination such as limonene and linalool, aldehyde transcine, tannin, coumarin, and resin. It was indicated that these compounds can decline the insulin resistance $(14,22,23)$. Considering the beneficial effects of both herbal medicines on the plasma levels of FBS and insulin in the groups that received both extracts together, the level of insulin and FBS decreased significantly.

Assessment of lipid profile in this research revealed that administration of A. graveolens and C. zeylanicum extracts had considerably positive effects on regulation of plasma lipids profile level such as a reduction in serum level of LDL, TG, and cholesterol and an increase in the level of HDL. Tuzcu et al also found that the C. zeylanicum extract could inhibit the hyperlipidemia and increase the level of HDL (24). Furthermore, Kooti et al claimed that the A. graveolens extract could decrease the serum lipid and regulate the lipid profile (25). Given that the extract used in the current study had antioxidant properties and could reduce the oxidative stress, administration of both extracts declined the plasma level of cholesterol, LDL, and
TG and enhanced the level of HDL.

In this research, it was observed that the PCOS caused damage in ovarian tissue such as the production of cystic follicles and atretic body in the ovary and also a decline in the number of normal follicles. Moreover, assessing oxidative stress marker in the ovarian tissue showed that the PCOS led to the increment in lipid peroxidation and also a reduction in SOD and GPX level in the ovarian tissue. These phenomena are possible originated from hyperandrogenism that led to the production of cystic follicles and stepped down in the normal follicles (26). Several studies demonstrated that enhancing the reactive oxygen species (ROS) level in the tissue resulted in oxidative stress and tissue damage (16,27-31). Mannera et al, for example, showed that the PCOS enhanced the atretic follicles while reducing the healthy antral follicles (32). Apparently, treatment with A. graveolens and C. zeylanicum extracts could protect the ovarian tissue from the oxidative damage by their antioxidant property. Besides, the ability of these extract in the prevention of hyperinsulinemia and hyperandrogenism may be a justification for these results.

\section{Conclusions}

Based on the results it was revealed that A. graveolens and C. zeylanicum extracts significantly influenced the regulation of serum levels of FBS, insulin, TG, LDL, and cholesterol in the palliation of the PCOS complications. Moreover, administering this extract separately and together could protect the ovarian tissue damages induced by the PCOS.

\section{Ethical Issues}

The present study was approved by the Ethics Committee of Tabriz University of Medical Sciences (TBZMED) under the ethical code of IR.TBZMED.VCR.REC.1397.22. 


\section{Conflict of Interests}

There is no conflict of interests.

\section{Financial Support}

This study was financially supported by Tabriz University of Medical Sciences (TBZMED), Tabriz, Iran.

\section{Acknowledgments}

The authors would like to thank the Deputy of Research and Technology of Tabriz University of Medical Sciences (TBZMED), for the financial support of this research.

\section{References}

1. Jafarisani M, Masoomikarimi M, Kazemi SS, Mirzaeidelaviz S, Naderi Z, Ahmadi R. Effect of Thymus vulgaris ethanol extract, on serum total antioxidant in experimental induced poly cystic ovarian syndrome (PCOs) rats. International Journal of Health Studies. 2016;2(1):30-4. doi:10.22100/ ijhs.v2i1.97

2. Zheng Q, Li Y, Zhang D, et al. ANP promotes proliferation and inhibits apoptosis of ovarian granulosa cells by NPRA/ PGRMC1/EGFR complex and improves ovary functions of PCOS rats. Cell Death Dis. 2017;8(10):e3145. doi:10.1038/ cddis.2017.494

3. Prelevic GM. Insulin resistance in polycystic ovary syndrome. Curr Opin Obstet Gynecol. 1997;9(3):193-201.

4. Martikainen H, Salmela P, Nuojua-Huttunen S, et al. Adrenal steroidogenesis is related to insulin in hyperandrogenic women. Fertil Steril. 1996;66(4):564-570.

5. Morin-Papunen LC, Koivunen RM, Ruokonen A, Martikainen HK. Metformin therapy improves the menstrual pattern with minimal endocrine and metabolic effects in women with polycystic ovary syndrome. Fertil Steril. 1998;69(4):691-696.

6. Diamanti-Kandarakis E, Mitrakou A, Raptis S, Tolis G, Duleba AJ. The effect of a pure antiandrogen receptor blocker, flutamide, on the lipid profile in the polycystic ovary syndrome. J Clin Endocrinol Metab. 1998;83(8):26992705. doi:10.1210/jcem.83.8.5041

7. Abtahi-Eivari SH, Moghimian M, Soltani M, et al. The effect of Galega officinalis on hormonal and metabolic profile in a rat model of polycystic ovary syndrome. Int J Womens Health Reprod Sci. 2018;6(3):276-282. doi:10.15296/ ijwhr.2018.46

8. Khaki A, Farnam A, Badie AD, Nikniaz H. Treatment effects of onion (Allium cepa) and Ginger (Zingiber officinale) on sexual behavior of rat after inducing an antiepileptic drug (lamotrigine). Balkan Med J. 2012;29(3):236-242. doi:10.5152/balkanmedj.2012.045

9. Shoorei H, Khaki A, Ainehchi N, et al. Effects of Matricaria chamomilla extract on growth and maturation of isolated mouse ovarian follicles in a three-dimensional culture system. Chin Med J (Engl). 2018;131(2):218-225. doi:10.4103/0366-6999.222324

10. Arentz S, Abbott JA, Smith CA, Bensoussan A. Herbal medicine for the management of polycystic ovary syndrome (PCOS) and associated oligo/amenorrhoea and hyperandrogenism; a review of the laboratory evidence for effects with corroborative clinical findings. BMC Complement Altern Med. 2014;14:511. doi:10.1186/1472-
6882-14-511

11. Fu N, Wang $\mathrm{Q}$, Shen HL. De novo assembly, gene annotation and marker development using Illumina paired-end transcriptome sequences in celery (Apium graveolens L.). PLoS One. 2013;8(2):e57686. doi:10.1371/ journal.pone.0057686

12. Popovic M, Kaurinovic B, Trivic S, Mimica-Dukic N, Bursac M. Effect of celery (Apium graveolens) extracts on some biochemical parameters of oxidative stress in mice treated with carbon tetrachloride. Phytother Res. 2006;20(7):531537. doi:10.1002/ptr.1871

13. Ching LS, Mohamed S. Alpha-tocopherol content in 62 edible tropical plants. J Agric Food Chem. 2001;49(6):31013105.

14. Dashti-R MH, Qane MD, Shefaie F, Nazemian Yazdu M, Bagheri SM. Comparative effect of cinnamon essential oil, diclofenac and morphine on acute and chronic pain in mice. Int J Med Laboratory. 2016;3(2):92-103.

15. Roushangar L, Rad JS. Ultrastructural alterations and occurrence of apoptosis in developing follicles exposed to low frequency electromagnetic field in rat ovary. Pak J Biol Sci. 2007;10(24):4413-4419.

16. Soltani M, Moghimian M, Abtahi-Eivari SH, Shoorei H, Khaki A, Shokoohi M. Protective effects of Matricaria chamomilla extract on Torsion/ detorsion-induced tissue damage and oxidative stress in adult rat testis. Int J Fertil Steril. 2018;12(3):242-248. doi:10.22074/ijfs.2018.5324

17. Zadehmodarres S, Heidar Z, Razzaghi Z, Ebrahimi L, Soltanzadeh K, Abed F. Anti-mullerian hormon level and polycystic ovarian syndrome diagnosis. Iran J Reprod Med. 2015;13(4):227-230.

18. Fazelian S, Rouhani MH, Bank SS, Amani R. Chromium supplementation and polycystic ovary syndrome: A systematic review and meta-analysis. J Trace Elem Med Biol. 2017;42:92-96. doi:10.1016/j.jtemb.2017.04.008

19. Gutierrez RMP, Juarez VA, Sauceda JV, Sosa IA. In vitro and in vivo antidiabetic and antiglycation properties of Apium graveolens in type 1 and 2 diabetic rats. Int J Pharmacol. 2014;10(7):368-379. doi:10.3923/ijp.2014.368.379

20. Lin LZ, Lu S, Harnly JM. Detection and quantification of glycosylated flavonoid malonates in celery, Chinese celery, and celery seed by LC-DAD-ESI/MS. J Agric Food Chem. 2007;55(4):1321-1326. doi:10.1021/jf0624796

21. Pinent M, Castell A, Baiges I, Montagut G, Arola L, Ardevol A. Bioactivity of flavonoids on insulin-secreting cells. Compr Rev Food Sci Food Saf. 2008;7(4):299-308. doi:10.1111/j.1541-4337.2008.00048.x

22. Qin B, Panickar KS, Anderson RA. Cinnamon: potential role in the prevention of insulin resistance, metabolic syndrome, and type 2 diabetes. J Diabetes Sci Technol. 2010;4(3):685-693. doi:10.1177/193229681000400324

23. Chen L, Sun P, Wang T, et al. Diverse mechanisms of antidiabetic effects of the different procyanidin oligomer types of two different cinnamon species on $\mathrm{db} / \mathrm{db}$ mice. J Agric Food Chem. 2012;60(36):9144-9150. doi:10.1021/ jf3024535

24. Tuzcu Z, Orhan C, Sahin N, Juturu V, Sahin K. Cinnamon polyphenol extract inhibits hyperlipidemia and inflammation by modulation of transcription factors in high-fat diet-fed rats. Oxid Med Cell Longev. 2017;2017:1583098. doi:10.1155/2017/1583098

25. Kooti W, Ali-Akbari S, Asadi-Samani M, Ghadery H, 
Ashtary-Larky D. A review on medicinal plant of Apium graveolens. Adv Herb Med. 2015;1(1):48-59.

26. De Leo V, la Marca A, Ditto A, Morgante G, Cianci A. Effects of metformin on gonadotropin-induced ovulation in women with polycystic ovary syndrome. Fertil Steril. 1999;72(2):282-285.

27. Soltani M, Moghimian M, Abtahi H, Shokoohi M. The protective effect of Matricaria chamomilla extract on histological damage and oxidative stress induced by Torsion/Detorsion in adult rat ovary. Int J Womens Health Reprod Sci. 2017;5(3):187-192. doi:10.15296/ijwhr.2017.34

28. Ameli M, Hashemi MS, Moghimian M, Shokoohi M. Protective effect of tadalafil and verapamil on testicular function and oxidative stress after torsion/detorsion in adult male rat. Andrologia. 2018;50(8):e13068. doi:10.1111/ and.13068

29. Khaki A, Fathiazad F, Nouri M, et al. Anti-oxidative effects of citro flavonoids on spermatogenesis in rat. Afr J Pharm Pharmacol. 2011;5(6):721-725. doi:10.5897/AJPP11.277

30. Khaki A. Evaluation effects of quercetin on liver apoptosis in streptozotocin-induced diabetic rat. J Med Plant. 2009;1(29):70-78.

31. Moghimian M, Abtahi-Evari SH, Shokoohi M, Amiri M, Soltani M. Effect of Syzygium aromaticum (clove) extract on seminiferous tubules and oxidative stress after testicular torsion in adult rats. Physiol Pharmacol. 2017;21(4):343350.

32. Manneras L, Cajander S, Lonn M, Stener-Victorin E. Acupuncture and exercise restore adipose tissue expression of sympathetic markers and improve ovarian morphology in rats with dihydrotestosterone-induced PCOS. Am J Physiol Regul Integr Comp Physiol. 2009;296(4):R11241131. doi:10.1152/ajpregu.90947.2008

(c) 2019 The Author (s); This is an open-access article distributed under the terms of the Creative Commons Attribution License (http://creativecommons.org/licenses/by/4.0), which permits unrestricted use, distribution, and reproduction in any medium, provided the original work is properly cited. 